\title{
KINEMATICS AND CHEMISTRY OF HALO SUBSTRUCTURES: THE VICINITY OF THE VIRGO OVERDENSITY
}

\author{
Andrew R. Casey, Stefan C. Keller, and Gary Da Costa \\ Research School of Astronomy \& Astrophysics, Australian National University, Mount Stromlo Observatory, via Cotter Rd, Weston, ACT 2611, Australia \\ Received 2011 August 25; accepted 2012 January 31; published 2012 March 14
}

\begin{abstract}
We present observations obtained with the Anglo-Australian Telescope's 2dF wide field spectrograph AAOmega of K-type stars located within a region of the sky which contains the Virgo Overdensity and the leading arm of the Sagittarius Stream. On the basis of the resulting velocity histogram, we isolate halo substructures in these overlapping regions including Sagittarius and previously discovered Virgo groups. Through comparisons with $\mathrm{N}$-body models of the Galaxy-Sagittarius interaction, we find a tri-axial dark matter halo is favored and we exclude a prolate shape. This result is contradictory with other observations along the Sagittarius leading arm, which typically favor prolate models. We have also uncovered K-giant members of Sagittarius that are notably more metal-poor $(\langle[\mathrm{Fe} / \mathrm{H}]\rangle=-1.7 \pm 0.3 \mathrm{dex})$ than previous studies. This suggests a significantly wider metallicity distribution exists in the Sagittarius Stream than formerly considered. We also present data on five carbon stars which were discovered in our sample.
\end{abstract}

Key words: Galaxy: halo - Galaxy: structure - stars: kinematics and dynamics

Online-only material: color figures

\section{INTRODUCTION}

The proportion of substructure recently uncovered in the Galaxy has highlighted the crucial involvement accretion has played in the formation of the Milky Way (see Helmi 2008, and references therein). Properties of these stellar structures allow us to probe the formation mechanisms and history of the Galaxy. Recent studies (Carollo et al. 2007, 2010) have suggested that multiple evolutionary paths are required for galaxy formation to reconcile observational evidence, although this is a subject of ongoing debate (Schönrich et al. 2011; Beers et al. 2012). Regardless, the dissipation-less merging paradigm is widely accepted and consistent with favored cold dark matter $(\Lambda \mathrm{CDM})$ cosmology models. Through the examination of ongoing accretion events in the Milky Way and fossils from previous mergers we can trace the evolution of the outer most regions of the Galaxy (e.g., Helmi \& White 2001).

Accretion is at least partly (e.g., Starkenburg et al. 2009), if not entirely responsible for the formation of the stellar halo. Bell et al. (2008) compared Sloan Digital Sky Survey (SDSS; York et al. 2000) data to galaxy formation simulations using different dark halos and found that observations are consistent with the stellar halo being entirely formed by hierarchical merging of accreted satellites (see also Xue et al. 2011).

Unquestionably, the most prominent ongoing accretion event within the Milky Way is that of the Sagittarius (Sgr) dwarf Spheroidal (dSph) galaxy. Originally discovered by Ibata et al. (1994) as a comoving group of K- and M-type giants, the tidal tails of Sgr circle our Galaxy. As such they have been extensively traced with red-clump stars (Majewski et al. 1999), carbon stars (Totten \& Irwin 1998; Ibata et al. 2001), RR Lyrae stars (Ivezić et al. 2000; Vivas et al. 2005; Keller et al. 2008; Watkins et al. 2009; Prior et al. 2009a), A-type stars (Newberg et al. 2003), blue horizontal branch (BHB) stars (Ruhland et al. 2011), and $\mathrm{K} / \mathrm{M}$ giants (Majewski et al. 2003; Yanny et al. 2009; Keller et al. 2010). Tracers originating from the host system can be unequivocally identified with spatial and kinematical information because they remain dynamically cold, and are identifiable as kinematic substructures long after they are stripped from their progenitor (for example, Ibata \& Lewis 1998; Helmi \& White 1999).

Stellar tracers within these tidal tails are kinematically sensitive to the galactic potential. This has led various groups to model the Sgr interaction with different dark matter profiles. Martínez-Delgado et al. (2004) traced the Northern leading arm and found a near spherical or oblate $(q \approx 0.85)$ dark matter halo best represented the observed debris, coinciding with the findings of Ibata et al. (2001). In contrast, Helmi (2004) found evidence in the Sgr leading debris that most favored a prolate $(q=1.25)$ halo. Vivas et al. (2005) found that either a prolate or spherical model of Helmi (2004) would fit their RR Lyrae observations, rather than those of an oblate model. Johnston et al. (2005) later pointed out that no prolate model can reproduce the orbital pole precession of the Sgr debris but an oblate potential could. Law et al. (2005, hereafter LJM05) performed simulations using data of the Sgr debris from the Two Micron All Sky Survey (2MASS) catalog and found that the kinematics of leading debris was best fit by prolate halos, whereas the trailing debris typically favored oblate halos. Prior et al. (2009b) reached a similar conclusion.

Belokurov et al. (2006) found an apparent bifurcation within the Sgr debris which Fellhauer et al. (2006) argued can only result from a dark halo having a near spherical shape. Law et al. (2009) introduced a tri-axial model with a varying flattening profile $q$, which replicates the orbital precession seen and matches kinematic observations of the Sgr debris. However, Law \& Majewski (2010, hereafter LM10) concede this may be a purely numerical solution as tri-axial halos are dynamically unstable. They emphasize that more kinematic measurements in other regions of the Sgr stream are required.

After the Sgr stellar stream, the Virgo Overdensity (VOD) is arguably the next most significant substructure within our Galaxy. The first overdensity in the vicinity of the VOD was observed as a group of RR Lyrae stars by the QUEST survey (Vivas et al. 2001). The collaboration later named this the "12.4 clump" (Zinn et al. 2004). The broad nature of the 


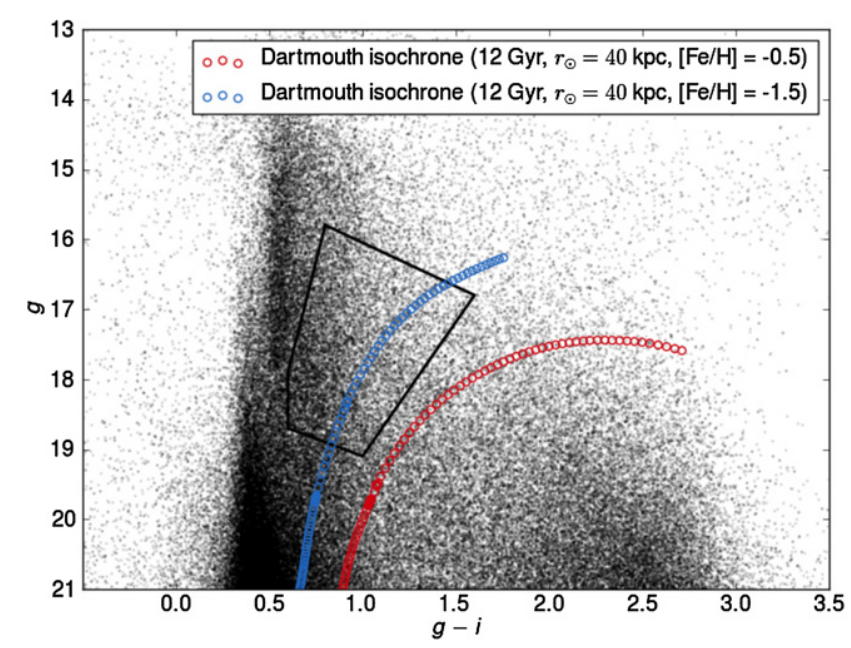

Figure 1. CMD of our observed regions from SDSS DR7, overlaid with the color selection criterion used to target K giants. Appropriate Dartmouth isochrones (Dotter et al. 2008) are shown for Sgr debris at a distance of $r_{\odot}=40 \mathrm{kpc}$ (Belokurov et al. 2006).

(A color version of this figure is available in the online journal.)

VOD was later uncovered from the SDSS catalog as a diffuse overdensity of main-sequence turnoff stars centered at $r_{\odot} \sim$ $18 \mathrm{kpc}$ (which Newberg et al. 2002, dubbed as S297+63-20.5). The nomenclature on the substructure names within this region is varied, however in this paper when referring to the VOD we are discussing the spatial overdensity of stars within the region, separate from any detected comoving groups.

The difficulty arises in accurately distinguishing the VOD as there are multiple substructures along this line of sight. Duffau et al. (2006) took observations of BHB and RR Lyrae within the " 12 h 4 clump" and found a common velocity of $V_{\mathrm{GSR}}=99.8 \mathrm{~km} \mathrm{~s}^{-1}$ with $\sigma_{v}=17.3 \mathrm{~km} \mathrm{~s}^{-1}$. It should be noted that the dispersion in kinematics measured by Duffau et al. (2006) is essentially their velocity precision, so the substructure may possess a much smaller kinematic dispersion. This comoving group was coined the Virgo Stellar Stream (VSS) to differentiate it from the broad spatial overdensity. This distinction from the VOD was somewhat strengthened with new distance measurements which placed the VOD centroid at $r_{\odot}=16 \mathrm{kpc}$ (Juric et al. 2008; Keller 2010), and the VSS $3 \mathrm{kpc}$ further away (Duffau et al. 2006). Although, when considered in the light of systematic and observational uncertainties, this is of marginal significance. Additionally, Jurić et al. (2008) suggest that the VOD may extend between $r_{\odot}=6$ to $20 \mathrm{kpc}$, which further complicates the matter of distance separation.

The relationship between the VSS and the S297+63-20.5 overdensity is still unclear. Newberg et al. (2007) found a kinematic signature at $V_{\mathrm{GSR}}=130 \pm 10 \mathrm{~km} \mathrm{~s}^{-1}$ for members of the VOD/S297+63-20.5, which is extremely close to the VSS peak. The VSS and S297+63-20.5 are coincident in space, but their velocity difference has not yet been reconciled. The distance measurements between S297+63-20.5 and the VSS are similar enough $(\sim 1 \mathrm{kpc})$ within probable distance uncertainties for Newberg et al. (2007) and Prior et al. (2009b) to infer they are part of the same structure. Newberg et al. (2007) estimate a distance to $\mathrm{S} 297+63-20.5$ of $r_{\odot}=18 \mathrm{kpc}$ from $g_{0}=20.5$ turnoff stars, but they concede the structure is likely dispersed along the line of sight as the color-magnitude diagram (CMD) for this region does not demonstrate a tight sequence. Certainly this region of sky, aptly coined the "Field of Streams" by Belokurov et al. (2006), is complex territory.
Table 1

Observed Fields

\begin{tabular}{lccc}
\hline \hline Field & $\begin{array}{c}\alpha \text {-center } \\
(\mathrm{J} 2000.0)\end{array}$ & $\begin{array}{c}\delta \text {-center } \\
(\mathrm{J} 2000.0)\end{array}$ & $\begin{array}{c}\text { Field } \\
\text { Configurations }\end{array}$ \\
\hline $\mathrm{A}$ & 120000 & +000000 & 1 \\
$\mathrm{~B}$ & 122000 & -010000 & 2 \\
$\mathrm{C}$ & 124000 & -020000 & 3 \\
$\mathrm{D}$ & 125600 & -024200 & 6 \\
\hline
\end{tabular}

Photometric studies are inadequate to fully untangle this region. Kinematics are essential to identify comoving groups that are distinct from the general halo field. Chemical information is vital to accurately distinguish these substructures and understand their origins. However, very few studies have directly investigated metallicities for these stars. In this paper we present spectroscopic observations of K giants in this region. Sgr stream kinematics are used to probe the shape of the dark matter halo in the Galaxy. We report both the velocities and metallicities of these giants in an effort to help untangle this accretiondominated region.

Target selection methodology is outlined in the next section which is followed with details regarding the observations. Techniques used to separate $\mathrm{K}$ giants from dwarfs are discussed in Section 4, and our analysis procedure for kinematics (Section 5) and metallicities (Section 6) follows. A discussion of substructures is outlined in Section 7, and in Section 8 we report the carbon stars discovered in our sample. In Section 9, we conclude with some final remarks and critical interpretations.

\section{TARGET SELECTION}

When the presence of a stellar substructure is uncovered, $\mathrm{K}$ giants provide excellent candidates for spectroscopic follow-up. They allow for precise radial velocities and chemical abundances. In order to specifically target $\mathrm{K}$ giants we have chosen candidates within the color selection box shown in Figure 1, taken from the SDSS DR7 catalog. This selection box favors a metal-poor population at Sgr stream-like distances ( $\sim 40 \mathrm{kpc})$ but will also contain similarly metal-poor stars at VOD/VSS distances $(\sim 20 \mathrm{kpc})$ without being significantly biased. Field dwarfs are expected to contaminate the sample due to their similarity in colors. Although $\mathrm{K}$ dwarfs are difficult to distinguish photometrically we can spectroscopically separate these through the equivalent width (EW) of the gravity-sensitive Mg I triplet lines at 5167.3, 5172.7, and 5183.6 $\AA$ (see Section 4).

\section{OBSERVATIONS}

Our targets were observed over two runs using AAOmega on the $3.9 \mathrm{~m}$ Anglo-Australian Telescope at Siding Springs Observatory in New South Wales, Australia. AAOmega is a doublebeam, multi-object (392) fiber-fed spectrograph covering a two degree field of view. The targets were observed in normal visitor mode in 2009 April. Throughout all observations, sufficient sky fibers $(\sim 30)$ were allocated to ensure optimal sky subtraction. In total 3453 science targets were observed across four fields within the VOD/Sgr region, as outlined in Table 1. Multiple configurations were observed for most fields to permit measurements of bright $(i<16)$ and faint $(i>16)$ stars, as well as repeat observations on a subset of stars.

The beam was split into the red and blue arms using the $5700 \AA$ Aichroic. The $580 \mathrm{~V}$ grating in the blue arm yields spectra between 370 and $580 \mathrm{~nm}$, with a resolution of $R=1300$. In the 
red arm we used the 1000I grating $(R=4400)$ which spans the spectral range from 800 to $950 \mathrm{~nm}$. This coverage includes the Ca II NIR Triplet (CaT), which is used for radial velocities and metallicities. Science targets on each configuration were limited to $1.5 \mathrm{mag}$ in range to minimize scattered-light cross talk between fibers. Globular clusters NGC 5024, 5053, and 5904 were observed as radial velocity and metallicity standards.

The data were reduced using the $2 \mathrm{DFDR}^{1}$ pipeline. After being flat-fielded, the fibers were throughput calibrated and the sky spectrum was subtracted using the median flux of the dedicated sky fibers. Wavelength calibration was achieved from arc lamp exposures taken between each set of science fields. Multiple object frames were combined to assist with cosmic ray removal.

\section{DWARF/GIANT SEPARATION}

When discussing our data with respect to stellar streams and substructures within the halo, we are referring only to K-type giants. Dwarfs that fall within our apparent magnitude limit are not sufficiently distant to probe halo substructures. Our resolution is adequate such that the $\mathrm{Mg}$ I triplet lines can be individually measured and used to discriminate against dwarfs.

A grid of synthetic spectra has been generated to quantitatively establish a suitable giant/dwarf separation criterion. The grid was generated using Castelli \& Kurucz (2004) model atmospheres with MOOG $^{2}$ and the line list of Kurucz \& Bell (1995). Spectra were also generated using stellar parameters for the Sun and Arcturus. The strength of the Mg I lines was tuned to match both the Solar and Arcturus atlases of Hinkle et al. (2003). Girardi et al. (2004) isochrones have been used to translate our de-reddened $g-i$ color range to effective temperature. Reddening is accounted for using the Schlafly \& Finkbeiner (2011) corrected dust maps of Schlegel et al. (1998) assuming a Fitzpatrick (1999) dust profile where $R_{V}=3.1$. The corresponding effective temperature region ranges from 3900 to $5200 \mathrm{~K}$ and is stepped at $25 \mathrm{~K}$ intervals. We have assumed typical K-type surface gravities of $\log g=2$ for giants and $\log g=4.5$ for dwarfs. Metallicities of $[\mathrm{Fe} / \mathrm{H}]=-0.5,-1.5$, and -2.5 were considered for both surface gravities.

All synthetic spectra were mapped onto the same wavelength intervals before the flux was convolved with a Gaussian kernel of $3.03 \AA$ to match our $580 \mathrm{~V}$ observations. Mg I line strengths for our observations and synthetic spectra are shown against $g-i$ in Figure 2. As expected, there is an overlap of $\mathrm{Mg} \mathrm{I}$ strengths between metal-rich "giants" $(\log g=2)$ and metalpoor "dwarfs" $(\log g=4.5)$. However, we do not expect metal-poor dwarfs to be a principle contaminant due to their intrinsically low luminosities and comparative rarity.

Using our synthetic grid, we have varied the slope and offset of our separation line to assess the effectiveness in differentiating giants from dwarfs. A linear rule has been adopted to differentiate the populations and maximize giant recoverability with minimal contamination. Using the rule,

$$
\mathrm{EW}_{\mathrm{Mg}}<3(g-i)_{o}+0.5
$$

we identify 185 giants in our observed fields. An analysis of our 185 giant candidates revealed three stars with high proper motions (PPMXL Catalog; Roeser et al. 2010), all of which lay close to our dwarf/giant separation line. This suggests that they

\footnotetext{
1 http://www.aao.gov.au/AAO/2df/aaomega

2 http://www.as.utexas.edu/ chris/moog.html
}

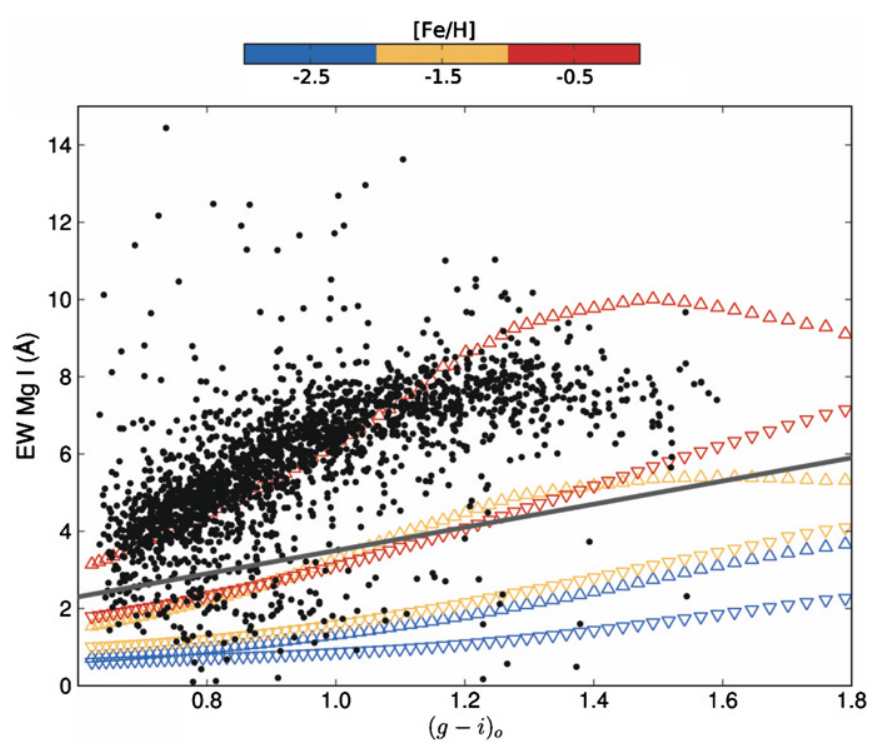

Figure 2. Sum of the Mg I triplet EWs for observations and synthetic spectra shown against the Sloan $g-i$ color. Synthetic spectra are shown for $\log g=2$ $(\nabla)$ and $\log g=4.5(\triangle)$, and points are colored by metallicity. Our dwarf/giant separation line is shown (solid).

(A color version of this figure is available in the online journal.)

are dwarfs, and they have been excluded. Some possible giant targets were also discarded due to insufficient signal-to-noise ratio, or because the $\mathrm{Mg}$ I triplet fell on bad columns of the detector. All efforts were made to minimize these losses. The distilled giant sample size is 178 stars. All identified K-giant stars in our distilled sample had no proper motion detectable above measurement errors.

\section{RADIAL VELOCITIES}

The CaII triplet absorption lines at 8498.0, 8542.1, and $8662.1 \AA$ have been used to measure radial velocities. These lines are strong, and easily identifiable in red giant branch (RGB) stars even at low resolution. Observed spectra have been cross-correlated with a synthetic spectrum of a typical $\mathrm{K}$ giant $\left(T_{\text {eff }}=4500 \mathrm{~K}, \log g=2,[\mathrm{Fe} / \mathrm{H}]=-1.5\right)$ to measure radial velocities. Heliocentric corrections were also applied. Radial velocity measurements made of the standard stars in our globular clusters agree (within $3 \mathrm{~km} \mathrm{~s}^{-1}$ ) with the catalog of Harris (1996, 2011 edition). Further, a number of our targets were observed on multiple fields, which allows us to calculate the internal measurement error. The differences between multiple measurements of the same target were calculated and they form a half-normal distribution with a $\mathrm{HWHM}=3.58 \mathrm{~km} \mathrm{~s}^{-1}$.

In order to compare our kinematic results in a homogenous manner, we have translated our heliocentric velocities to a galactocentric frame. We have adopted the circular velocity of the local standard of rest (LSR) at the Sun as $220 \mathrm{~km} \mathrm{~s}^{-1}$ (Kerr \& Lynden-Bell 1986) and accounted for the Sun's peculiar velocity to the LSR by using $16.5 \mathrm{~km} \mathrm{~s}^{-1}$ toward $l=53^{\circ}, b=25^{\circ}$ (Mihalas \& Binney 1981). The corrected line-of-sight velocity is then given by

$$
\begin{aligned}
V_{\mathrm{GSR}}= & V_{\mathrm{OBS}}+220 \sin l \cos b+16.5 \\
& \times[\sin b \sin 25+\cos b \cos 25 \cos (l-53)],
\end{aligned}
$$

where $V_{\mathrm{OBS}}$ is the heliocentric-corrected observed line-of-sight velocity. A caveat to this reference transformation is that other 
authors in the literature have used slightly different formulae to transpose their kinematics to a galactocentric frame. This will result in possible systematic shifts in velocities between authors of up to $\sim 11 \mathrm{~km} \mathrm{~s}^{-1}$.

\section{METALLICITIES}

We have measured metallicities for our giants using the strength of the $\mathrm{Ca}$ triplet lines. This technique was originally empirically described for individual stars in globular clusters (Armandroff \& Da Costa 1991). A spectroscopic analysis using VLT/FLAMES observations of RGB stars from composite populations led Battaglia et al. (2008) to conclude that a calibrated $\mathrm{CaT}-[\mathrm{Fe} / \mathrm{H}]$ relationship can be confidently used in composite stellar populations (see also Rutledge et al. 1997; Starkenburg et al. 2010). The caveat to this technique is that a luminosity (specifically $V-V_{\mathrm{HB}}$ ) is required for calibration, and we have to assume a $V_{\mathrm{HB}}$ luminosity here. Johnson $V$-band magnitudes for our giants were calculated from SDSS ugriz photometry using Jester et al. (2005) transformations. The weaker third $\mathrm{Ca}$ triplet line is more susceptible to noise and residual sky-line contamination (Tolstoy et al. 2001; Battaglia et al. 2008). Consequently, only the strongest two CaT lines (8542 and $8662 \AA$ ) have been used to form a reduced EW $\left(W^{\prime}\right)$ such that

$$
\begin{gathered}
\sum W=\mathrm{EW}_{8542}+\mathrm{EW}_{8662} \\
W^{\prime}=\sum W+0.64( \pm 0.02)\left(V-V_{\mathrm{HB}}\right)
\end{gathered}
$$

and the metallicity linearly varies with $W^{\prime}$, where

$$
[\mathrm{Fe} / \mathrm{H}]_{\mathrm{CaT}}=(-2.81 \pm 0.16)+(0.44 \pm 0.04) W^{\prime} .
$$

Using this calibration our globular cluster standard stars (with known $V_{\mathrm{HB}}$ magnitudes) have metallicities that match well with the Harris (1996) catalog (2011 edition). Only K giants within the valid calibration range $\left(0>V-V_{\mathrm{HB}}>-3\right)$ were considered for metallicities. We assume that we have two dominant substructures present in our observations; the leading arm of Sgr and the VOD (see Section 7.1). The Sgr stream dominates our negative $V_{\mathrm{GSR}}$ population, and our positive kinematic space primarily comprises VOD and VSS members. As such we have separated our population at $V_{\mathrm{GSR}}=0 \mathrm{~km} \mathrm{~s}^{-1}$ into two samples with assumed $V_{\mathrm{HB}}$ magnitudes. Although this introduces a (known) systemic effect, it is a required assumption to estimate the metallicity distribution of each population.

The horizontal branch magnitude assumed for the VSS/VOD has been ascertained from previous RR Lyrae studies. As RR Lyrae stars sit on the horizontal branch, the $V_{\mathrm{HB}}$ is taken as the $V$-band median of four Prior et al. (2009b) and three Duffau et al. (2006) VSS members to yield $\langle V\rangle=17.09$. This combined value precisely matches the mean reported by Duffau et al. (2006). In our K-giant sample with positive galactocentric velocities, we assume $V_{\mathrm{HB}}=17.09$ which implies these stars are at a distance of $\sim 20 \mathrm{kpc}$.

As the distance to the Sgr debris varies greatly throughout the halo, the horizontal branch magnitude varies with the position along the stream. Through CMD comparisons with the Sgr core (Bellazzini et al. 2006), Belokurov et al. (2006) determined distances along the two branches of the Sgr bifurcation. Revised distance measurements by Siegel et al. (2007, as tabulated in Table 1 of LM10) have been crucial in constraining dark halo models. Our observations lay on the edge of Branch A (Figure 5).

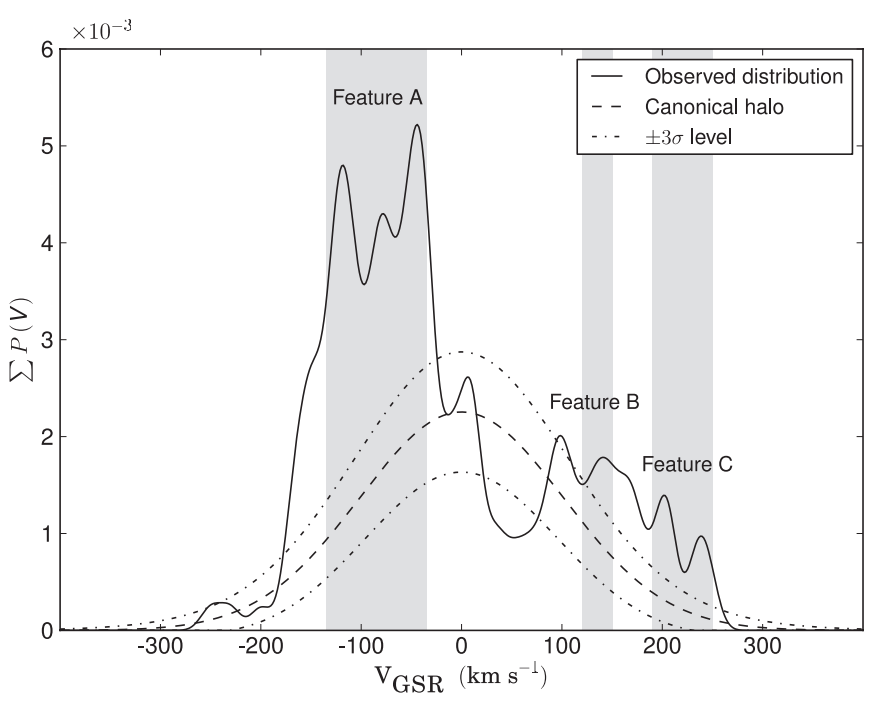

Figure 3. Generalized histogram of $V_{\mathrm{GSR}}$ for our $178 \mathrm{~K}$-type giants, highlighting the substructure present. The Gaussian represents the halo contribution; it is normalized such that the integral equals the number of observed stars excluding those outside a $2.5 \sigma$ excess. Significant $(>3 \sigma)$ kinematic deviations from the smooth distribution are appropriately grouped and labeled.

We have assumed distances to these stars by interpolating their position along a cubic spline fitted to published distances (Siegel et al. 2007). Recent distance measurements of the stream by Ruhland et al. (2011) match the stream distances used here within the uncertainties. The horizontal branch magnitude $V_{\mathrm{HB}}$ is calculated using this assumed distance and the known luminosity of RR Lyrae stars $\left(M_{V}=+0.69\right.$; Tsujimoto et al. 1998). Typical $V_{\mathrm{HB}}$ magnitude derived for the Sgr members is 18.7 , corresponding to a distance of $\sim 41 \mathrm{kpc}$. Reddening is accounted for using the Schlegel et al. (1998) maps with Schlafly \& Finkbeiner (2011) corrections as described in Section 4. Uncertainties in distance are not interpolated; they are taken as the largest published uncertainty of the closest neighboring data points, and this is propagated through to our reported metallicity uncertainties. The kinematics and metallicities derived for our giant sample are discussed in the following section.

\section{DISCUSSION}

\subsection{Substructure Identification}

A significant kinematic deviation from a canonical halo population signifies a comoving group. There are multiple substructures along our line of sight. These features are identified first and discussed separately. We have represented our galactocentric velocities with a generalized histogram (Figure 3) to quantitatively compare the observed stellar kinematics against the hypothesis that the parent distribution can be described by a canonical halo where $\mu=0 \mathrm{~km} \mathrm{~s}^{-1}$ and $\sigma=101.6 \mathrm{~km} \mathrm{~s}^{-1}$ (Sirko et al. 2004).

The generalized histogram represents each data point with a Gaussian kernel of an equal deviation. As our internal kinematic errors between multiple measurements summate to a halfnormal distribution with an FWHM of $7.16 \mathrm{~km} \mathrm{~s}^{-1}$, we have opted for a sigma value of $10 \mathrm{~km} \mathrm{~s}^{-1}$ for the generalized histogram in Figure 3 to avoid undersmoothing. The most significant $(>3 \sigma)$ kinematic peaks have been labeled Features $\mathrm{A}, \mathrm{B}$, and $\mathrm{C}$. Features $\mathrm{A}$ and $\mathrm{C}$ are our most significant structures, and the importance of Feature B becomes more evident through Monte Carlo simulations. 

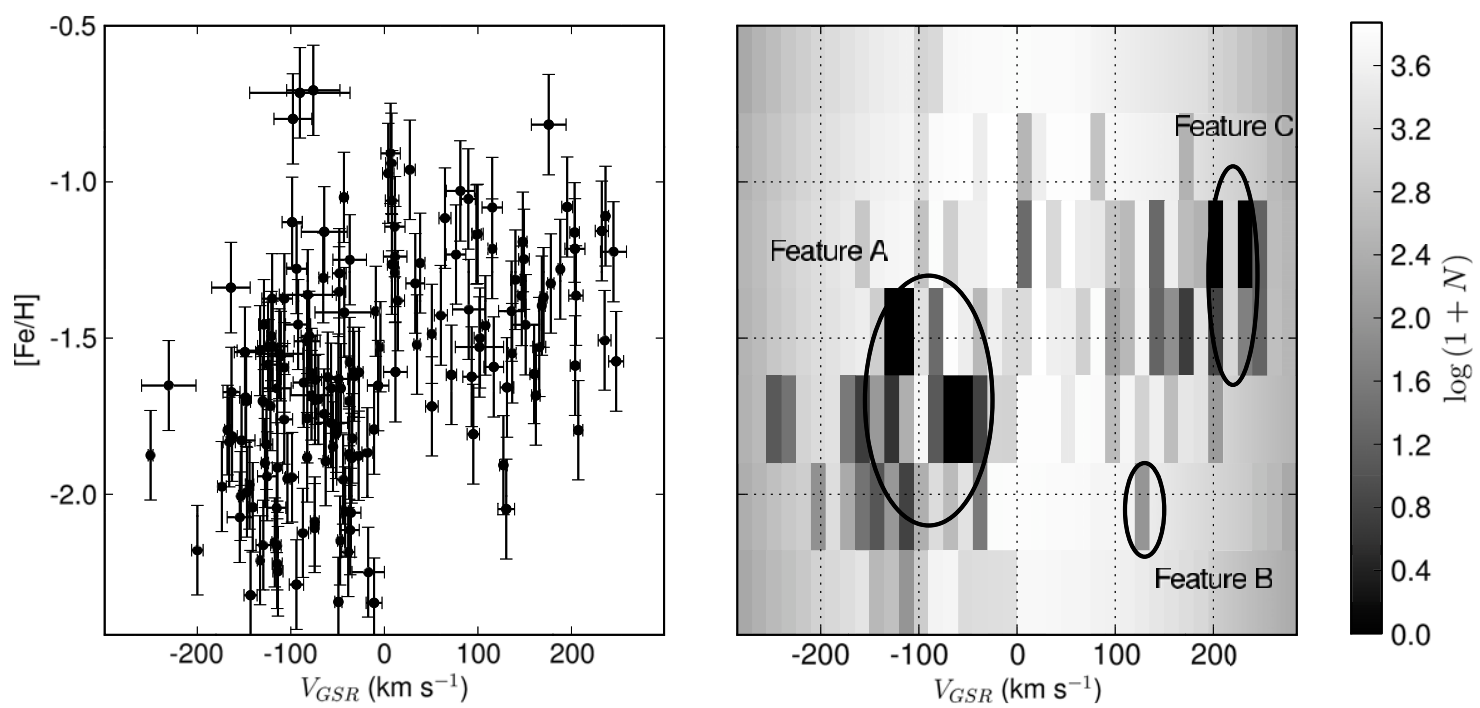

Figure 4. Observed data for the 178 giant star sample (left panel). Monte Carlo simulation results illustrating the number of times simulations could reproduce our observed data in the equivalent multi-dimensional bin (right panel). Significant features discussed in the text are labeled.

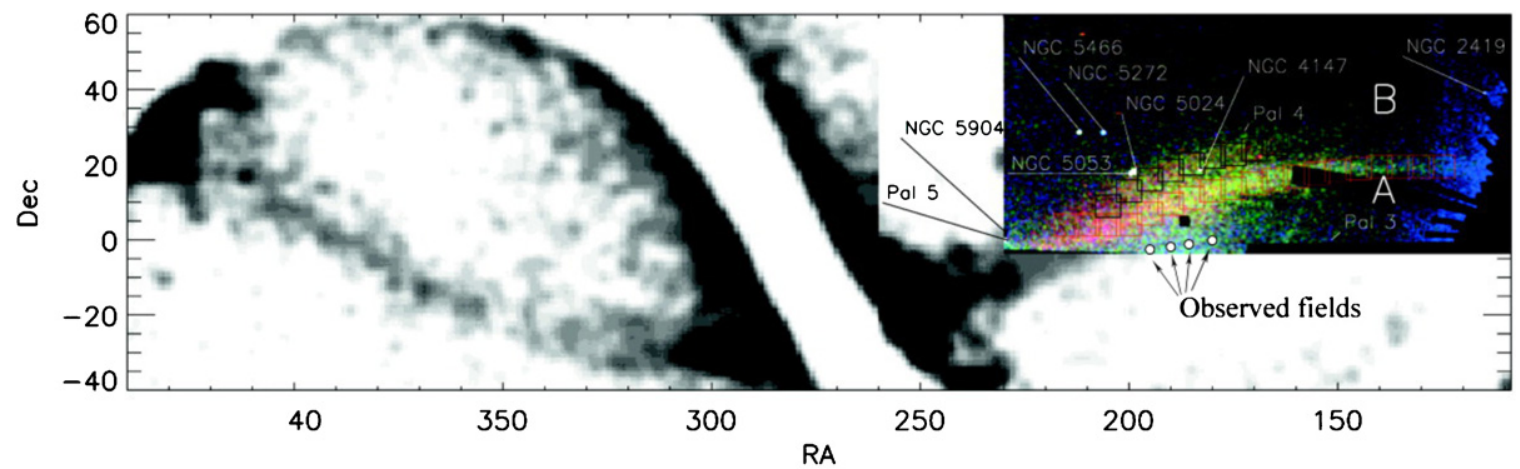

Figure 5. Observed fields are outlined upon a panoramic view of the Sgr stream, to demonstrate our field locations in context with the Sgr stream. This plot is an adaptation of Figure 2 in Belokurov et al. (2006), which uses the 2MASS M-giant sample of Majewski et al. (2003).

(A color version of this figure is available in the online journal.)

Velocities and metallicities of our $\mathrm{K}$ giants, shown in the left panel of Figure 4, were binned into grid blocks of $15 \mathrm{~km} \mathrm{~s}^{-1}$ and 0.3 dex-roughly twice the error in each dimension. To interpret these data a population of 178 stars was randomly drawn from a simulated halo with canonical kinematics. Metallicities were randomly assigned using the observed halo Metallicity Distribution Function (MDF) of Ryan \& Norris (1991). Each simulated population was binned identically to our observed sample. Simulation grid blocks with counts equal to or exceeding stars in the equivalent observed grid block were noted and summed after 10,000 simulations. Results from our Monte Carlo simulations are illustrated in Figure 4 (right). The identified Features $\mathrm{A}, \mathrm{B}$, and $\mathrm{C}$ in our results were consistently significant when the grid was midpoint offset in both dimensions, except for the grid block centered at $\sim 185 \mathrm{~km} \mathrm{~s}^{-1}$ and $\sim-1.4$ dex, which has consequently been left unlabeled.

Substructure becomes statistically significant when the observed grid blocks are rarely replicated in Monte Carlo simulations. Specifically, the number of members in Feature A was never reproduced in some grid bins. This feature is well in excess of the halo and has a wide spread in kinematics. Chou et al. (2007) mapped the Sgr debris across the sky using $\mathrm{K} / \mathrm{M}$ giants and found galactocentric velocity signatures between $-205 \mathrm{~km} \mathrm{~s}^{-1}$ to $-31 \mathrm{~km} \mathrm{~s}^{-1}$ in this region. As such we attribute this wide, significant kinematic peak as the leading arm of the Sgr tidal tail. Feature A is discussed in the next section. Another feature where grid blocks were never reproduced in our simulations was Feature C. This feature may also be attributed to Sgr debris and is discussed further in Section 7.4.

The clump of stars in the velocity bin centered on $V_{\mathrm{GSR}} \approx$ $130 \mathrm{~km} \mathrm{~s}^{-1}$ and $[\mathrm{Fe} / \mathrm{H}] \approx-2$ was replicated only $\sim 1 \%$ in 10,000 simulations. We attribute these stars to the VSS, as they are coincident in spatial position, velocity, and metallicity with previously reported values of the VSS obtained by F turnoff/BHB stars (Newberg et al. 2007) and RR Lyrae stars (Prior et al. 2009b). Feature B is discussed in Section 7.3.

\subsection{Feature A-Sagittarius Debris}

Our observed fields are overlaid upon a panoramic projection of the Sgr stream and the "Field of Streams" (Belokurov et al. 2006) in Figure 5. This is a crowded region of globular clusters, substructures, and overlapping stellar streams, primarily populated by the Sgr Northern leading arm. Simply from a spatial perspective, we expect the Sgr debris to dominate our data. Although the VOD is present, it is much more diffuse.

\subsubsection{Comparing Sagittarius Debris to Dark Matter Halo Models}

In order to investigate the spatial coverage and kinematics of our Sgr members, we have compared our data with the 

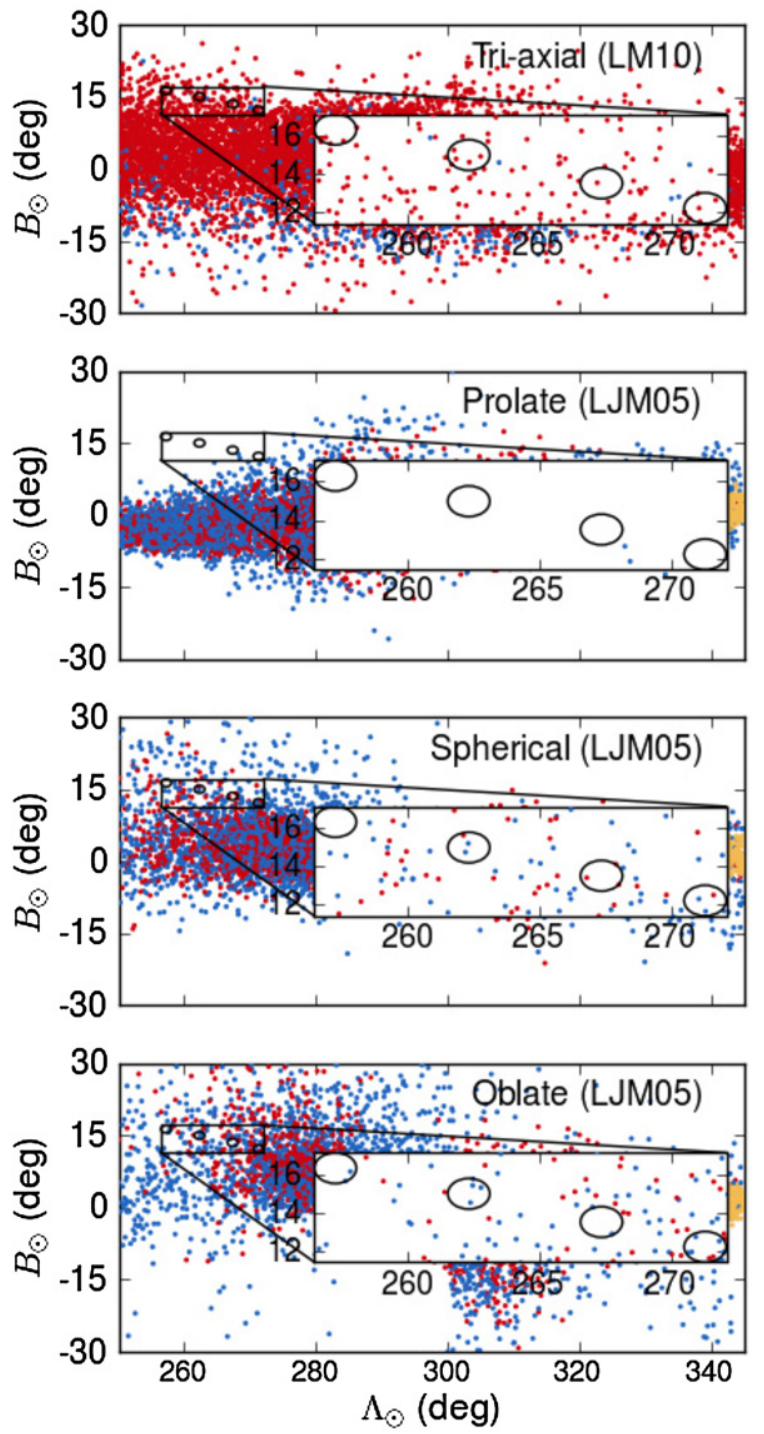

Figure 6. Our fields and observed bounds are shown with the simulated particles from multiple $N$-body simulations by LJM05 and LM10. These particles are distance restricted (up to $60 \mathrm{kpc}$ ), and color coded by their peri-centric passage (yellow: most recent passage, red: previous passage, blue: oldest observable passage).

(A color version of this figure is available in the online journal.)

constant flattening spheroidal models of LJM05, and the more recent tri-axial model of LM10. The simulated data output from these models is readily available online, ${ }^{3}$ and the released models have the best-fitting parameters for each dark halo shape (prolate, spherical, oblate, and tri-axial). These are the only simulations available which make use of an all-sky data set; the 2MASS M-giant sample. We have not considered particles farther than $60 \mathrm{kpc}$ to match realistic observation limitations. The $10^{5}$ model Sgr debris particles from their simulations are represented along the best-fit great-circle $\left(\Lambda_{\odot}, B_{\odot}\right)$ where the longitudinal coordinate $\Lambda_{\odot}$ is zero in the direction of the Sgr core and increases along the trailing debris. For comprehensive details of the simulations the reader is referred to the papers of Law et al. (2005) and Law \& Majewski (2010).

The Northern leading arm of Sgr is particularly sensitive both kinematically and spatially to the shape of the Milky Way dark halo (Figure 6). The spherical, oblate, and tri-axial

\footnotetext{
3 http://www.astro.virginia.edu/ $\sim \mathrm{srm} 4 \mathrm{n} / \mathrm{Sgr} /$
}

models predict the Sgr stream to pass directly through our fields, whereas the prolate model predicts only the edge of the stream near this region and no particles directly in our fields. Spatially, the prolate model predicts the Sgr stream to pass much lower in latitude $\left(B_{\odot}\right)$ than our observed fields. When we extend a rectangle bounded by the edges of our fields (as per the zoomed insets in Figure 6) a mere two model particles are found. The $10^{5}$ model particles are proportional to the stream density so the lack of model particles implies a negligible stream concentration in our observed region. Observationally, when the number of field configurations is accounted for our observed Sgr K giants are uniformly distributed across the fields. If this prolate halo model is a true representation of the Sgr debris, this does not exclude the potential of finding some Sgr debris in our fields. However, it does imply that if the LJM05 prolate model is an accurate representation of the dark halo then Sgr would not be the dominant population-contrary to our preferred interpretation of the observations.

In comparison, the tri-axial, spherical, and oblate models predict varying amounts of debris from previous peri-centric passages in our fields. A kinematic comparison against our observations is necessary to evaluate model predictions. As the prolate model has only two simulated particles within our extended bounds there is no qualitative kinematic comparison to be made for this model, therefore the prolate model is excluded from further kinematic analysis.

The number of predicted particles observable differs between models. Consequently, the kinematics for each model have been represented as a generalized histogram (Figure 7). For consistency all simulation output and observed data have been convolved with a Gaussian kernel of $10 \mathrm{~km} \mathrm{~s}^{-1}$; the sigma used for the previous generalized histogram in Figure 3. Predicted distances and peri-centric ages are shown, demonstrating that debris from multiple passages are predicted along the line of sight. A much tighter velocity sequence is predicted by the triaxial model than the constant flattening models. This is because the tri-axial model predicts a peri-center almost precisely in our observable region, whereas the spherical and oblate models predict a large wrap along the line of sight, resulting in a wide spread of distances and velocities.

Predicted distances are consistent with what we would expect from our observations. The median luminosities of our giants are $g \sim 17.5$, so for the tri-axial model particles at $45 \mathrm{kpc}$ this corresponds to an absolute magnitude of $M_{g} \sim-0.8$; quite reasonable for a $\mathrm{K}$ giant. Similarly for the spherical and oblate models, typical distances of $\sim 25 \mathrm{kpc}$ will yield luminosities of $M_{g} \sim+0.5$ which is also reasonable.

The spherical and oblate models also predict close-by debris with extremely negative galactocentric velocities. This signature is not represented in our data. The lowest observed velocity is $\sim-250 \mathrm{~km} \mathrm{~s}^{-1}$ and we have only two observations less than $V_{\mathrm{GSR}}<-200 \mathrm{~km} \mathrm{~s}^{-1}$. In contrast, the spherical/oblate models predict velocities well below $V_{\mathrm{GSR}}<-300 \mathrm{~km} \mathrm{~s}^{-1}$. These predicted highly negative velocities were also not found in $\mathrm{Sgr}$ RR Lyrae observations taken by Prior et al. (2009a). If the dark matter potential is well represented by either an oblate or spherical halo, then this discrepancy must be reconciled.

The oblate and spherical model also illustrate a similar signature at $r_{\odot} \sim 12 \mathrm{kpc}$, with particle velocities ranging between $-100 \mathrm{kms}^{-1}<V_{\mathrm{GSR}}<0 \mathrm{~km} \mathrm{~s}^{-1}$. This is the edge of a predicted crossing-point between different wraps of the stream, which occurs at $\sim 12 \mathrm{kpc}$. These particles (and the positive kinematic signature around $\sim 20 \mathrm{kpc}$ in the oblate model), are 

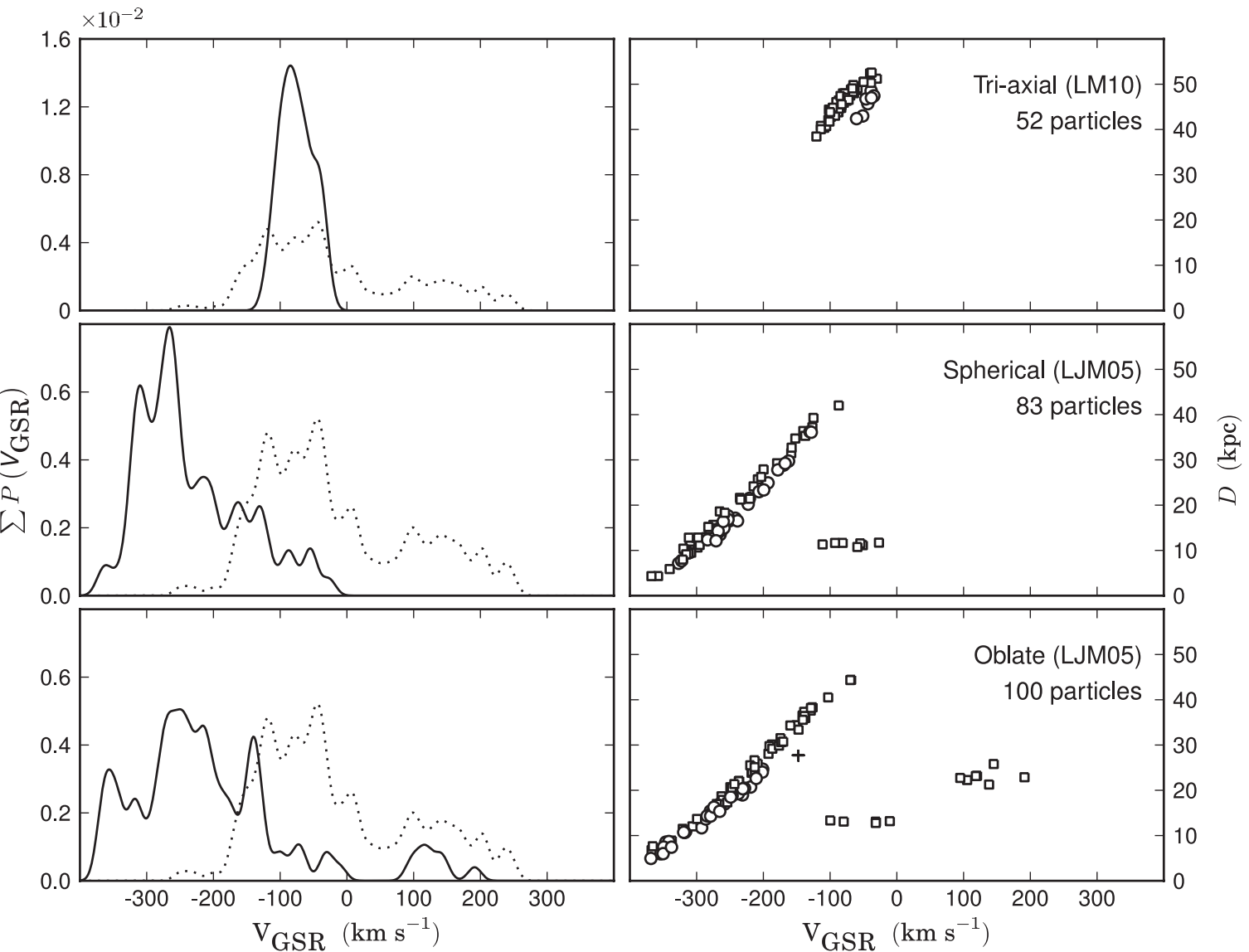

Figure 7. Generalized histogram (left) of the galactocentric rest-frame velocities in our sample (dotted line) compared to the velocities (solid line) from the $N$-body models of LJM05, LM10 of particles within the same spatial coverage and distance range (up to $r_{\odot} \sim 60 \mathrm{kpc}$ ) of our observed sample. Heliocentric distances for particles used to generate each velocity histogram are shown on the right. Particles are marked by their peri-centric passage. A plus $(+)$ denotes debris from the current peri-centric passage by Sgr, circles ( $)$ mark the previous passage, and squares $(\square)$ represent debris from two previous passages. The prolate model from LJM05 is not considered in this plot (see text).

relatively minor signatures when compared to the Northern arm feature. If these signatures are present in our observed fields, their relatively low density compared to the Northern arm feature would prevent them from appearing as significant.

Although the predicted velocity distribution is narrower than what we observe, the LM10 tri-axial dark halo model best fits our observations. The observed sample broadens most prominently toward more negative galactocentric velocities at $\sim-200 \mathrm{~km} \mathrm{~s}^{-1}$, considerably higher than oblate/spherical model predictions. Halo contamination at highly negative velocities is likely to be small. One reconciliation for this discrepancy between our observations and the tri-axial model may lie in the workings of the tri-axial model itself. Unlike other models considered, the tri-axial model does not reproduce the observed bifurcation in the Sgr stream (Belokurov et al. 2006). Although observationally untested, it is reasonable to suggest a bifurcation may result from a significant kinematic disruption. Such an effect could result in a broader kinematic distribution-similar to what we have observed-which is most notable at the stream edges.

There is further work required through observations and simulations to reconcile kinematic discrepancies. Typical examinations of the leading arm debris usually favor prolate halos and evidence along the trailing arm typically favors oblate halos (Helmi 2004; Martínez-Delgado et al. 2004; Law et al. 2005). Kinematic predictions of the LM10 tri-axial model reasonably match our observations, whereas the prolate model has been excluded as significant debris is not predicted in this edge of the stream. These observations along the Northern leading arm are the first which are not reproducible with the current prolate model of LJM05, contrary to previous groups who have surveyed closer to the leading arm debris.

\subsubsection{A Metal-poor Population Uncovered in Sagittarius Debris}

Many groups have found the population of the Sgr core to possess a mean $[\mathrm{Fe} / \mathrm{H}] \sim-0.5$ dex (Cacciari et al. 2002; Bonifacio et al. 2004; Monaco et al. 2005). The observed metallicity gradient along the stream suggests that as the host circles the Milky Way the older, more metal-poor stars are preferentially stripped from the progenitor (Chou et al. 2007; Keller et al. 2010). In this region of the stream very few Sgr member metallicities have been reported. Vivas et al. (2005) found a mean metallicity of $\langle[\mathrm{Fe} / \mathrm{H}]\rangle=-1.77$ from spectra of 16 RR Lyrae stars along a nearby region of the Sgr leading arm. Similarly, Prior et al. (2009a) found $\langle[\mathrm{Fe} / \mathrm{H}]\rangle=-1.79 \pm 0.08$ for 21 type $a b$ RR Lyrae stars in the region. This is somewhat expected since only the oldest, most metal-poor stars can form RR Lyraes.

Investigating the MDF of the Sgr debris requires an unbiased sample. Generally, K giants are excellent stellar candidates for such investigations as all stars go through this evolutionary phase whereas $\mathrm{M}$ giants are consistently metal-rich. If we apply the metallicity gradient found by Keller et al. (2010, from M giants) to these observed $\mathrm{K}$ giants, we would expect an abundance mean near $\sim-1.2$ dex in this observed region. The metallicity distribution for our entire negative $V_{\mathrm{GSR}}$ sample is shown in 


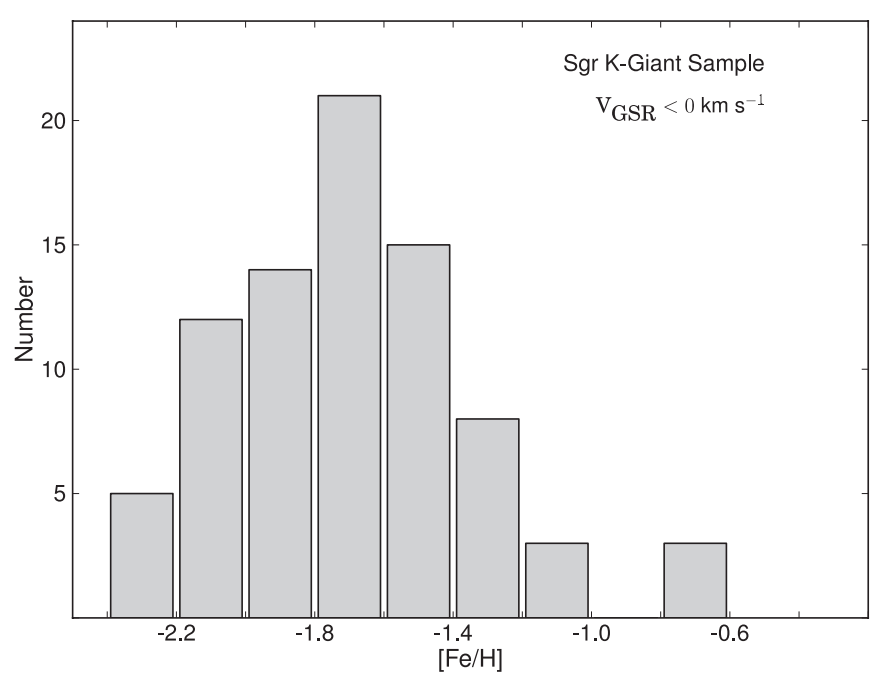

Figure 8. Metallicity histograms for K-giant members in our negative galactocentric velocity sample, which is largely populated by Sgr debris from the Northern leading arm.

Figure 8 and illustrates a metal-poor population. The mean of our distribution is $\langle[\mathrm{Fe} / \mathrm{H}]\rangle=-1.7 \pm 0.3$ dex. If we include only the stars attributed to Feature $\mathrm{A}$ (as defined by the shaded region in Figure 3, between $-140 \mathrm{kms}^{-1}<V_{\mathrm{GSR}}<-30 \mathrm{~km} \mathrm{~s}^{-1}$ ) this value increases by only $0.04 \mathrm{dex}$; well within observational uncertainties. As a comparison, in a sample of metal-rich biased M giants from the 2MASS data Chou et al. (2007) found a mean metallicity of $\langle[\mathrm{Fe} / \mathrm{H}]\rangle=-0.72$ dex for their best subsample in the Northern leading arm of Sgr. Although there may be some halo contamination in our sample, in general our more metalpoor Feature A members have higher negative velocities, which is expected for Sgr stream members in this region.

It is likely that our observed K-giant sample is biased toward more metal-poor members. Given a typical distance of $r_{\odot} \sim 40 \mathrm{kpc}$ for the Sgr debris in this region (Belokurov et al. 2006), a 12 Gyr old Dartmouth (Dotter et al. 2008) isochrone with a metallicity of $[\mathrm{Fe} / \mathrm{H}]=-1.5$ falls directly within our target selection window. The more metal-rich isochrone with $[\mathrm{Fe} / \mathrm{H}]=-0.5$ does not pass through our selection range. This is consistent with our observed metallicities for Sgr. Although this does not prohibit the possibility of more metal-rich members within our sample, it implies that our distribution is biased toward the metal-poor end of the MDF. The tail of our Sgr distribution extends from -0.71 to -2.35 dex.

\subsection{Feature B-The Virgo Stellar Stream}

Another substructure which was distinguished from the general overdensity of the VOD is the VSS (Duffau et al. 2006). Several RR Lyrae stars were noted with a common velocity of $V_{\mathrm{GSR}}=130 \mathrm{~km} \mathrm{~s}^{-1}$ (Newberg et al. 2007; Prior et al. 2009b). Prior et al. (2009b) also measured metallicities of stars with this kinematic signature and found a mean $[\mathrm{Fe} / \mathrm{H}]=-1.95 \pm 0.1$ for the VSS, although this is based on RR Lyrae stars and therefore is potentially biased to the oldest, most metal-poor populations. The RR Lyrae sample from Duffau et al. (2006) found an $\langle[\mathrm{Fe} / \mathrm{H}]\rangle=-1.86$ with a much larger abundance spread of $\sigma=0.40$, which was several times the relative uncertainty in individual values $\left(\sigma_{[\mathrm{Fe} / \mathrm{H}]}=0.08 \mathrm{dex}\right)$. This led Duffau et al. (2006) to infer the progenitor of the VSS was likely a dSph galaxy, as a large dispersion in $[\mathrm{m} / \mathrm{H}]$ indicates self-enrichment and implies the structure is a galaxy and not an isolated stellar cluster. Although some globular clusters are also now known to contain multiple populations and sizeable $[\mathrm{m} / \mathrm{H}]$ dispersions, globular clusters in general have a much smaller dispersion than that observed here. Similar to Duffau et al. (2006), we also observe an abundance spread within this kinematic bin including two $\mathrm{K}$ giants with mean kinematics and metallicities $\left(\left\langle V_{\mathrm{GSR}}\right\rangle=130 \pm 9 \mathrm{~km} \mathrm{~s}^{-1},\langle[\mathrm{Fe} / \mathrm{H}]\rangle=\right.$ $-2.0 \pm 0.16)$ matching those found by Prior et al. (2009b). Monte Carlo simulations reproduced these targets a mere $\sim 1 \%$ in 10,000 simulations-highlighting their significance.

High-resolution follow-up spectroscopy on these targets and other VSS candidates at higher metallicities in this kinematic range will provide crucial information about the origin of the VSS. Investigating $[\alpha / \mathrm{Fe}]$ ratios in these candidates can constrain the mass of the VSS progenitor and discern whether the host was indeed a dSph (Venn et al. 2004; Casetti-Dinescu et al. 2009). Future observations are planned.

\subsection{Feature C-Sagittarius Debris?}

The last significant kinematic substructure we identify in our data is Feature $\mathrm{C}$. There are two peaks identified here at $V_{\mathrm{GSR}}=200$ and $240 \mathrm{~km} \mathrm{~s}^{-1}$. These features cannot be explained by a smooth halo distribution. Newberg et al. (2007) identified a $4 \sigma$ peak at $V_{\mathrm{GSR}}=200 \mathrm{~km} \mathrm{~s}^{-1}$ in their sample of $\mathrm{F}$ turnoff stars centered on $(l, b)=\left(288^{\circ}, 62^{\circ}\right)$. Our nearest field to their plate (Field B) hosts only one star associated with this peak; most of our stars are located within our most populated Field D. Prior et al. (2009a) also noted stars in this kinematic range and compiled a list of authors who have similarly observed such peaks (see Sirko et al. 2004; Duffau et al. 2006; Starkenburg et al. 2009). Prior et al. (2009a) argue these stars may be associated with trailing debris of Sgr, as suggested by models (LJM05, LM10). Although this trailing debris would be much closer than the nearby visible leading arm, our observations support the interpretation by Prior et al. (2009a) as the metallicities are quite similar to the nearby Sgr debris.

This substructure has a range of metallicities. These metallicities were derived assuming a single distance modulus to the VOD/VSS. The abundance dispersion we observe is either representative, or these stars have a common metallicity and are dispersed along the line of sight. Unfortunately, neither can be positively excluded without further observations. If these peaks are associated with the trailing debris of Sgr debris, they are likely to be further away than the VOD. On our scale that would make these stars more metal-rich than shown in Figure 4. However, the nearest trailing debris particles predicted from any best-fitting dark halo potential that could explain this kinematic peak occur at $\sim \Lambda_{\odot}=310^{\circ}$; our observations range in longitude between $\Lambda_{\odot}=256^{\circ}-273^{\circ}$. Whether this substructure is separate from Sgr debris or not remains equivocal. Nevertheless, the apparent metallicities of the Feature $\mathrm{C}$ stars are not inconsistent with those of a Sgr population given our results for Feature A and those of Keller et al. (2010).

\section{CARBON STARS}

In our sample we have identified five carbon stars. Our color selection to select $\mathrm{K}$ giants overlaps with where we would expect to find carbon stars. Although these stars were not specifically targeted, their surface densities are so low $\left(\approx 1\right.$ per $50 \mathrm{deg}^{2}$; Green et al. 1994) that we would not expect them as a substantial contaminant. Although our sky coverage is small $\left(\sim 12.5 \mathrm{deg}^{2}\right)$, the observed carbon star spatial density is $\sim 20 \times$ higher than expected. All five carbon stars are recognizable by the presence of distinctive 4737 and $5165 \AA$ Swan $\mathrm{C}_{2}$ bands. 
Table 2

Properties of Carbon Stars Found in Our Sample

\begin{tabular}{|c|c|c|c|c|c|c|c|c|c|c|c|}
\hline SDSS Name $\mathrm{J}^{\mathrm{a}}$ & $M_{g}$ & $u-g$ & $g-r$ & $r-i$ & $i-z$ & $H-K$ & $J-H$ & $\begin{array}{c}\mu_{\alpha \cos \delta}{ }^{\mathrm{b}} \\
\left(\mathrm{mas}_{\mathrm{yr}} \mathrm{y}^{-1}\right)\end{array}$ & $\begin{array}{c}\mu_{\delta}{ }^{\mathrm{b}} \\
\left(\mathrm{mas}_{\mathrm{yr}} \mathrm{yr}^{-1}\right)\end{array}$ & $\begin{array}{c}V_{\mathrm{GSR}} \\
\left(\mathrm{km} \mathrm{s}^{-1}\right)\end{array}$ & $\begin{array}{c}\text { Likely } \\
\text { Dwarf? }\end{array}$ \\
\hline $121740.94-001839.5$ & 18.66 & 1.10 & 0.54 & 0.16 & 0.05 & $\ldots{ }^{\mathrm{c}}$ & $\ldots \mathrm{c}$ & $-3.0 \pm 5.4$ & $-18.0 \pm 5.4$ & $-71 \pm 34$ & Yes \\
\hline $121853.18-004628.4$ & 17.71 & 1.67 & 0.73 & 0.22 & 0.11 & 0.24 & 0.36 & $-3.7 \pm 4.5$ & $-0.5 \pm 4.5$ & $+176 \pm 9$ & $\cdots$ \\
\hline $122053.71-011709.5$ & 17.65 & 1.79 & 0.80 & 0.26 & 0.13 & 0.16 & 0.56 & $-7.3 \pm 4.3$ & $-3.9 \pm 4.3$ & $+31 \pm 11$ & $\cdots$ \\
\hline $125410.80-032744.0$ & 18.59 & 1.43 & 0.64 & 0.22 & 0.05 & 0.56 & 0.32 & $-21.1 \pm 5.0$ & $-7.7 \pm 5.0$ & $-41 \pm 9$ & Yes \\
\hline $125416.52-031437.6$ & 16.85 & 1.72 & 0.73 & 0.25 & 0.11 & -0.11 & 0.50 & $-19.8 \pm 4.2$ & $8.7 \pm 4.2$ & $+15 \pm 4$ & Yes \\
\hline
\end{tabular}

Notes.

a To keep with convention, positional information has been truncated. Full information available through the SDSS archive.

b Proper motions taken from the PPMXL Catalog (Roeser et al. 2010).

c No $J H K$ photometry is available for this object as it is fainter than the 2MASS survey limit.

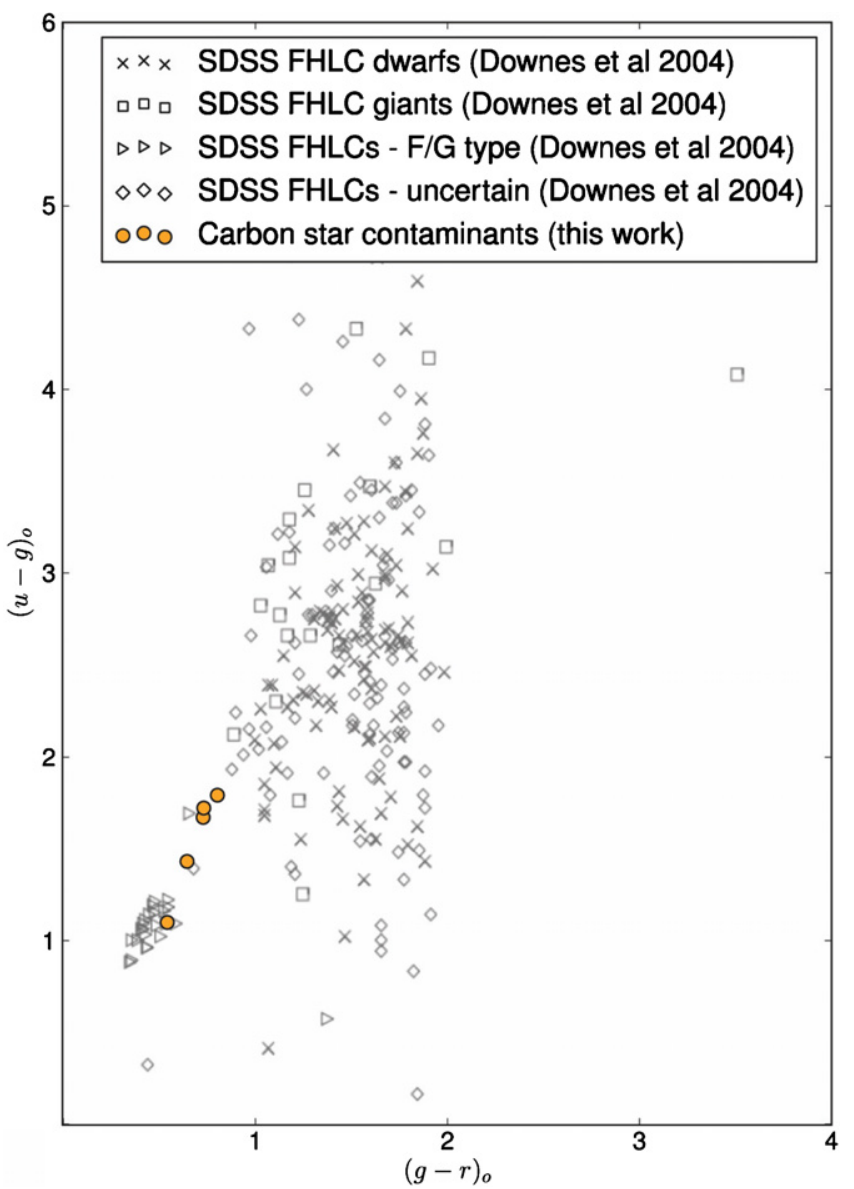

Figure 9. Sloan $u-g$ and $g-r$ colors for our carbon stars, and the identified carbon stars and classifications by Downes et al. (2004). Carbon stars identified in this work have been shaded for clarity.

(A color version of this figure is available in the online journal.)

Dwarf carbon stars exhibit a spectral signature which mimics that of a typical CH-type giant carbon star, however they have anomalous $J H K$ colors (Green et al. 1992) and high proper motions. The existence of the $4300 \AA \mathrm{CH} G$ band is representative of a $\mathrm{CH}$-type carbon star and is found in all five of our carbon stars. SDSS photometry for our stars match well with the F/G-type $\mathrm{CH}$ carbon stars identified by Downes et al. (2004), as seen in Figure 9. However, these targets were not spectroscopically observed in the SDSS follow-up survey SEGUE. Through comparisons with previous carbon-type star catalogs (Totten \& Irwin 1998; Downes et al. 2004; Goswami et al. 2010), the stars tabulated in Table 2 are previously unclassified carbon stars. This is largely because our objects are too faint to have been observed by previous spectroscopic carbon star surveys.

There is 2MASS photometry available for four of these stars. Of those, two stars (SDSS J125410.80-032744.0 and J125416.52-031437.6) exhibit anomalous JHK colors and significant $(>3 \sigma)$ proper motions (PPMXL; Roeser et al. 2010); characteristics of a dwarf carbon star. A third star also exhibits significant proper motion (SDSS J121740.94-001839.5), however $J H K$ photometry is unavailable. The two remaining stars in our sample have $J H K$ photometry characteristic of the $\mathrm{F} / \mathrm{G}$ type $\mathrm{CH}$ carbon stars found by Downes et al. (2004) in their Faint High-Latitude Carbon star survey (Figure 9), and do not exhibit significant proper motion-which strongly suggests they are not dwarfs (Green et al. 1994; Deutsch 1994).

\section{CONCLUSIONS}

We present spectroscopic observations of K-type stars in a region of the "Field of Streams," where significant substructure is present. We utilize the gravity-sensitive Mg I triplet to separate giants and dwarfs. Radial velocities and metallicities of $178 \mathrm{~K}$ giants have been determined. We have recovered kinematic substructure found by other authors, which cannot be explained by a smooth halo distribution. Highly negative velocity signatures match those expected by the Sgr stream debris, and we also identify a group of $\mathrm{K}$ giants with metallicities and kinematics which make them highly probable members of the VSS.

Stars in this region of the Sgr stream are kinematically sensitive to the shape of the Galactic dark halo. As such, we have compared our velocity distribution to the Sgr-Milky Way dark matter models of Law et al. (2005) and Law \& Majewski (2010). Typically, leading arm debris favor prolate models and trailing arm debris favor oblate models. A prolate dark halo predicts relatively little debris in our observed fields. If the spheroid is prolate and LJM05 present an accurate representation, Sgr debris would not be expected to dominate our sample even if we were $\Delta B_{\odot} \sim 10^{\circ}$ closer to the great-circle best fit. However, Sgr debris is our most significant kinematic structure observed. No single model perfectly represents our data, although we find the more recent tri-axial model (LM10) best represents our observed kinematics.

Observed metallicities for $\mathrm{K}$ giants in this region of the $\mathrm{Sgr}$ stream are notably lower than expected based on other Sgr samples, demonstrating the presence of a metal-poor population in the Sgr debris. Although isochrones indicate we are biased toward metal-poor members at these distances, these stars are unequivocally $\mathrm{Sgr}$ in origin. Metallicity gradients reported suggest a mean of $\langle[\mathrm{Fe} / \mathrm{H}]\rangle \sim-1.2$, whereas our population has $\langle[\mathrm{Fe} / \mathrm{H}]\rangle=-1.7 \pm 0.3$ dex. Previously reported abundances in 
this region have been ascertained from observing RR Lyrae stars (where the mean metallicity found was $\langle[\mathrm{Fe} / \mathrm{H}]\rangle \sim$ $-1.7 \mathrm{dex}$ ) or from observing $\mathrm{M}$ giants (where $\langle[\mathrm{Fe} / \mathrm{H}]\rangle \sim$ -0.7 dex was reported). In both scenarios the measurements are reasonable with what we would expect from each particular stellar population. Thus, the sample presented here is more representative of the complete MDF, and less susceptible to age or metallicity biases. These observations also demonstrate that the Sgr stream likely hosts a substantially larger abundance range than previously found, including a previously undetected metal-poor population of $\mathrm{K}$ giants.

A.R.C. acknowledges the financial support through the Australian Research Council Laureate Fellowship 0992131. S.K. and G.D.C. acknowledge the financial support from the Australian Research Council through Discovery Program DP0878137.

This publication makes use of data products from the Two Micron All Sky Survey, which is a joint project of the University of Massachusetts and the Infrared Processing and Analysis Center/California Institute of Technology, funded by the National Aeronautics and Space Administration and the National Science Foundation.

Funding for the SDSS and SDSS-II has been provided by the Alfred P. Sloan Foundation, the Participating Institutions, the National Science Foundation, the U.S. Department of Energy, the National Aeronautics and Space Administration, the Japanese Monbukagakusho, the Max Planck Society, and the Higher Education Funding Council for England. The SDSS Web site is http://www.sdss.org/.

The SDSS is managed by the Astrophysical Research Consortium for the Participating Institutions. The Participating Institutions are the American Museum of Natural History, Astrophysical Institute Potsdam, University of Basel, University of Cambridge, Case Western Reserve University, University of Chicago, Drexel University, Fermilab, the Institute for Advanced Study, the Japan Participation Group, Johns Hopkins University, the Joint Institute for Nuclear Astrophysics, the Kavli Institute for Particle Astrophysics and Cosmology, the Korean Scientist Group, the Chinese Academy of Sciences (LAMOST), Los Alamos National Laboratory, the Max-PlanckInstitute for Astronomy (MPIA), the Max-Planck-Institute for Astrophysics (MPA), New Mexico State University, Ohio State University, University of Pittsburgh, University of Portsmouth, Princeton University, the United States Naval Observatory, and the University of Washington.

\section{REFERENCES}

Armandroff, T. E., \& Da Costa, G. S. 1991, AJ, 101, 1329

Battaglia, G., Irwin, M., Tolstoy, E., et al. 2008, MNRAS, 383, 183

Beers, T. C., Carollo, D., Ivezić, Ž., et al. 2012, ApJ, 746, 34

Bellazzini, M., Correnti, M., Ferraro, F. R., Monaco, L., \& Montegriffo, P. 2006, A\&A, 446, L1

Bell, E. F., Zucker, D. B., Belokurov, V., et al. 2008, ApJ, 680, 295

Belokurov, V., Zucker, D. B., Evans, N. W., et al. 2006, ApJ, 642, L137

Bonifacio, P., Sbordone, L., Marconi, G., Pasquini, L., \& Hill, V. 2004, A\&A, 414, 503

Cacciari, C., Bellazzini, M., \& Colucci, S. 2002, in IAU Symp. 207, Extragalactic Star Clusters, ed. D. P. Geisler, E. K. Grebel, \& D. Minniti (Cambridge: Cambridge Univ. Press), 168

Carollo, D., Beers, T. C., Chiba, M., et al. 2010, ApJ, 712, 692

Carollo, D., Beers, T. C., Lee, Y. S., et al. 2007, Nature, 450, 1020

Casetti-Dinescu, D. I., Girard, T. M., Majewski, S. R., et al. 2009, ApJ, 701, L29
Castelli, F., \& Kurucz, R. L. 2004, arXiv:astro-ph/0405087

Chou, M., Majewski, S. R., Cunha, K., et al. 2007, ApJ, 670, 346

Deutsch, E. W. 1994, PASP, 106, 1134

Dotter, A., Chaboyer, B., Jevremović, D., et al. 2008, ApJS, 178, 89

Downes, R. A., Margon, B., Anderson, S. F., et al. 2004, AJ, 127, 2838

Duffau, S., Zinn, R., Vivas, A. K., et al. 2006, ApJ, 636, L97

Fellhauer, M., Belokurov, V., Evans, N. W., et al. 2006, ApJ, 651, 167

Fitzpatrick, E. L. 1999, PASP, 111, 63

Girardi, L., Grebel, E. K., Odenkirchen, M., \& Chiosi, C. 2004, A\&A, 422, 205 Goswami, A., Karinkuzhi, D., \& Shantikumar, N. S. 2010, MNRAS, 402, 1111

Green, P. J., Margon, B., Anderson, S. F., \& Cook, K. H. 1994, ApJ, 434, 319

Green, P. J., Margon, B., Anderson, S. F., \& MacConnell, D. J. 1992, ApJ, 400, 659

Harris, W. E. 1996, AJ, 112, 1487

Helmi, A. 2004, ApJ, 610, L97

Helmi, A. 2008, A\&AR, 15, 145

Helmi, A., \& White, S. D. M. 1999, MNRAS, 307, 495

Helmi, A., \& White, S. D. M. 2001, MNRAS, 323, 529

Hinkle, K., Wallace, L., Livingston, W., et al. 2003, in The Future of Cool-Star Astrophysics: 12th Cambridge Workshop on Cool Stars, Stellar Systems, and the Sun, ed. A. Brown, G. M. Harper, \& T. R. Ayres (Vol. 12; Boulder, CO: Univ. Colorado), 851

Ibata, R. A., Gilmore, G., \& Irwin, M. J. 1994, Nature, 370, 194

Ibata, R. A., \& Lewis, G. F. 1998, ApJ, 500, 575

Ibata, R., Lewis, G. F., Irwin, M., Totten, E., \& Quinn, T. 2001, ApJ, 551, 294

Ivezić, Ž., Goldston, J., Finlator, K., et al. 2000, AJ, 120, 963

Jester, S., Schneider, D. P., Richards, G. T., et al. 2005, AJ, 130, 873

Johnston, K. V., Law, D. R., \& Majewski, S. R. 2005, ApJ, 619, 800

Jurić, M., Ivezić, Ž., Brooks, A., et al. 2008, ApJ, 673, 864

Keller, S. C. 2010, PASA, 27, 45

Keller, S. C., Murphy, S., Prior, S., Da Costa, G., \& Schmidt, B. 2008, ApJ, 678, 851

Keller, S. C., Yong, D., \& Da Costa, G. S. 2010, ApJ, 720, 940

Kerr, F. J., \& Lynden-Bell, D. 1986, MNRAS, 221, 1023

Kurucz, R. L., \& Bell, B. 1995, Atomic Line Data, Kurucz CD-ROM No. 23 (Cambridge, MA: Smithsonian Astrophysical Observatory)

Law, D. R., Johnston, K. V., \& Majewski, S. R. 2005, ApJ, 619, 807

Law, D. R., \& Majewski, S. R. 2010, ApJ, 714, 229

Law, D. R., Majewski, S. R., \& Johnston, K. V. 2009, ApJ, 703, L67

Majewski, S. R., Siegel, M. H., Kunkel, W. E., et al. 1999, AJ, 118, 1709

Majewski, S. R., Skrutskie, M. F., Weinberg, M. D., \& Ostheimer, J. C. 2003, ApJ, 599, 1082

Martínez-Delgado, D., Gómez-Flechoso, M. Á., Aparicio, A., \& Carrera, R. 2004, ApJ, 601, 242

Mihalas, D., \& Binney, J. 1981, Science, 214, 829

Monaco, L., Bellazzini, M., Bonifacio, P., et al. 2005, A\&A, 441, 141

Newberg, H. J., Yanny, B., Cole, N., et al. 2007, ApJ, 668, 221

Newberg, H. J., Yanny, B., Grebel, E. K., et al. 2003, ApJ, 596, L191

Newberg, H. J., Yanny, B., Rockosi, C., et al. 2002, ApJ, 569, 245

Prior, S. L., Da Costa, G. S., \& Keller, S. C. 2009a, ApJ, 704, 1327

Prior, S. L., Da Costa, G. S., Keller, S. C., \& Murphy, S. J. 2009b, ApJ, 691, 306

Roeser, S., Demleitner, M., \& Schilbach, E. 2010, AJ, 139, 2440

Ruhland, C., Bell, E. F., Rix, H.-W., \& Xue, X.-X. 2011, ApJ, 731, 119

Rutledge, G. A., Hesser, J. E., \& Stetson, P. B. 1997, PASP, 109, 907

Ryan, S. G., \& Norris, J. E. 1991, AJ, 101, 1865

Schlafly, E. F., \& Finkbeiner, D. P. 2011, ApJ, 737, 103

Schlegel, D. J., Finkbeiner, D. P., \& Davis, M. 1998, ApJ, 500, 525

Schönrich, R., Asplund, M., \& Casagrande, L. 2011, MNRAS, 415, 3807

Siegel, M. H., Dotter, A., Majewski, S. R., et al. 2007, ApJ, 667, L57

Sirko, E., Goodman, J., Knapp, G. R., et al. 2004, AJ, 127, 899

Starkenburg, E., Helmi, A., Morrison, H. L., et al. 2009, ApJ, 698, 567

Starkenburg, E., Hill, V., Tolstoy, E., et al. 2010, A\&A, 513, A34

Tolstoy, E., Irwin, M. J., Cole, A. A., et al. 2001, MNRAS, 327, 918

Totten, E. J., \& Irwin, M. J. 1998, MNRAS, 294, 1

Tsujimoto, T., Miyamoto, M., \& Yoshii, Y. 1998, ApJ, 492, L79

Venn, K. A., Irwin, M., Shetrone, M. D., et al. 2004, AJ, 128, 1177

Vivas, A. K., Zinn, R., Andrews, P., et al. 2001, ApJ, 554, L33

Vivas, A. K., Zinn, R., \& Gallart, C. 2005, AJ, 129, 189

Watkins, L. L., Evans, N. W., Belokurov, V., et al. 2009, MNRAS, 398, 1757

Xue, X.-X., Rix, H.-W., Yanny, B., et al. 2011, ApJ, 738, 79

Yanny, B., Newberg, H. J., Johnson, J. A., et al. 2009, ApJ, 700, 1282

York, D. G., Adelman, J., Anderson, J. E., Jr., et al. 2000, AJ, 120, 1579

Zinn, R., Vivas, A. K., Gallart, C., \& Winnick, R. 2004, in ASP Conf. Ser. 327, Satellites and Tidal Streams, ed. F. Prada, D. Martinez Delgado, \& T. J. Mahoney (San Francisco, CA: ASP), 92 